\title{
Rendimiento académico, musical e inteligencias múltiples en alumnos preadolescentes de un centro específico de música
}

\author{
Amelia Barrientos Fernández, Roberto Sánchez Cabrero, y \\ Amaya Arigita García \\ Universidad Alfonso X el Sabio (España)
}

\begin{abstract}
Se realiza un estudio descriptivo correlacional que pretende evaluar la relación existente entre las inteligencias múltiples de alumnos de secundaria en un centro integrado de música y su rendimiento académico en materias musicales y generales, para comprobar si la estimulación de la inteligencia y el talento musical tienen repercusiones positivas sobre el rendimiento académico. Para ello, se ha aplicado en 28 alumnos de $1^{\circ}$ de secundaria el test de Inteligencia general y factorial de Yuste-Hernanz (1991), el Cuestionario de técnicas y hábitos de estudio (IHE) de Pozar (1994), el Cuestionario de IMCUIM (Aliaga et al., 2012) de inteligencias múltiples y se han recogido las calificaciones académicas de 2 evaluaciones continuas. Los resultados permiten concluir que, en general, los resultados de los estudiantes en las asignaturas han sido óptimos en ambas modalidades, la general y la musical. Se ha comprobado que estos estudiantes cuentan con un nivel intelectual por encima de la media y destacan por distinguirse en inteligencias múltiples, como la musical, la quinestésica y la interpersonal, por lo que se concluye que, no solo estimular la inteligencia musical no perjudica al rendimiento académico, sino que, muy posiblemente, contribuye a mejorarlo.
\end{abstract}

Palabras clave: Inteligencia, inteligencias múltiples, inteligencia musical, rendimiento académico, hábitos de estudio.

Academic, musical performance and multiple intelligences in pre-adolescent students of a specific music center. A correlational descriptive study is carried out to evaluate the relationship between the multiple intelligences of high school students in an integrated music center and their academic performance in musical and general matters, to check if the stimulation of intelligence and musical talent has positive influence in academic performance. To this end, the Yuste Hernanz general and factorial intelligence test (1991), Pozar's survey of techniques and study habits (IHE) (1994), the IMCUIM questionnaire (Aliaga et al. al., 2012) of multiple intelligences and the academic qualifications of 2 continuous evaluations have been collected. The results allow to conclude that, in general, the results of the students in the subjects have been optimal in both the general and the musical modalities. It has been proven that these students have an above-average intellectual level and stand out for distinguishing multiple intelligences, such as musical, kinesthetic and interpersonal, so it is concluded that not only stimulate musical intelligence does not harm the academic performance, but, quite possibly, contributes to improving it.

Keywords: Intelligence, multiple intelligences, musical intelligence, academic performance, study habits.

Correspondencia: Amelia Barrientos Fernández. Avenida Universidad, $n^{\circ} 1$, Edificio D, despacho 246. C.P.: 28691 Villanueva de la Cañada, Madrid (España). E-mail: abarrien@uax.es 
En el nuevo paradigma educativo, representado por las últimas leyes educativas (en España, LOE, 2006 y LOMCE, 2013), la formación en habilidades y desarrollo de las IM (en adelante IM) está siendo cada vez más relevante con el fin de capacitar a nuestro alumnado para la vida profesional y personal, cobrando así la misma consideración que la adquisición de contenidos. Por este motivo, cada vez son cada vez más numerosos los centros educativos que están implantando metodologías que faciliten la adquisición y desarrollo de dichas IM (Gardner, 2016).

En la actualidad, existen modelos que consideran la inteligencia desde otras perspectivas distintas a la cognitiva y no tanto desde una perspectiva puramente intelectual en que se valoraba simplemente el coeficiente intelectual. En bastantes modelos educativos, se está alentando a los profesores a llevar a cabo procesos didácticos mediante trabajos que ayuden a los estudiantes a desarrollar variadas inteligencias. Hay que tener cuenta que cada uno de los alumnos tiene un grado de adquisición diferente en las mencionadas inteligencias y, por ello, se hace necesario el apoyo y la orientación del profesor para facilitarles el desarrollo de todas y cada una de ellas, con el fin de lograr una formación completa que les facilite la puesta en práctica de los conocimientos en situaciones determinadas (Garagorri, 2007; López, 2017).

En la teoría de las IE, Gardner (1983) puso de manifiesto que la inteligencia no es una capacidad de nacimiento y está constituida desde las destrezas para resolver problemas hasta la habilidad para crear cosas nuevas. Sin embargo, no intentaba poner en entredicho la tenencia de una inteligencia general (que está constituida por la inteligencia lingüística y la lógico-matemática), sino de sustentar la presencia de otras. Gardner (2008) aportó datos empíricos de la existencia de distintas facultades cognitivas. Asimismo, plantea una hipótesis en la que define la capacidad intelectual como una compilación de distintas inteligencias que se apoyan unas en otras, interrelacionándose en algunos momentos y, en otros, destacan unas sobre otras, cuyo dominio depende de cada persona. Las IM están focalizadas en distintas partes del cerebro y se encuentran profundamente interrelacionadas entre sí y son motivadas y se acrecientan en situaciones apropiadas.

En muchas situaciones, esto no se produce de esta forma, ya que en el sistema educativo actual se siguen considerando como únicas y fundamentales las inteligencias tradicionales (matemática y lingüística). Vivas (2017) considera que se mejora el clima de aula y su organización cuando se proporcionan los medios y las estrategias adecuadas que permitan atender a los discentes de forma individualizada que les ayude a percibir sus puntos fuertes y débiles, y así lograr mejorar la calidad de la diversidad en el conjunto de estudiantes y, de ese modo, tomar medidas de ajuste con los alumnos que presentan problemas en su aprendizaje. De esta forma, se facilita un clima de aula más seguro y agradable en el que los alumnos estarán más abiertos a desarrollar su capacidad de aprender. 
Debemos recordar que entre las IM existe una fuerte relación que, en muchas ocasiones, es complicada porque cualquier persona a la hora de hacer una tarea suele hacer uso de más de una de las inteligencias y de forma simultánea (Ferrándiz et al., 2006). Cada individuo puede ejercitar distintas pericias en una misma inteligencia, como ocurre por ejemplo con un deportista que es muy hábil practicando su deporte, pero a la vez puede estar muy capacitado para tocar un instrumento musical (Ferrándiz et al., 2006; Gardner, 2016). Guzmán y Castro (2017) sostienen que todas las inteligencias se pueden fortalecer y además se debe llevar a cabo desde las menos desarrolladas. En este sentido, Andreu y Godall-Castell (2012) abogaron por la relevancia que tiene la educación musical como instrumento para mejorar la capacidad matemática.

Entre todos los talentos, la inteligencia musical es la que se ejercita de manera más precoz y está muy condicionada por el contexto en el que se desarrollen los individuos y cómo se estimule su crecimiento (Barrientos-Fernández et al., 2019). Asimismo, depende de diversos factores como los genéticos, las habilidades involucradas (capacidad de oído, de creación y reproducción musical, de análisis, etc.). Esta habilidad se focaliza en el hemisferio derecho, concretamente en el lóbulo frontal del cerebro y destaca porque se tiene agilidad para discriminar sonidos, para reconocer ruidos normales, para distinguir sonidos en función de su dirección e intensidad y, en la música, diferenciar entre la melodía, el ritmo, el tono, la frecuencia y el timbre y disociar sonidos en grupos musicales (Gardner, 2016; Koelsch, 2012). La inteligencia musical se puede interconectar con las demás IM, pero especialmente con la cinestésico-corporal y la lógico-matemática, no obstante, es muy independiente, y además requiere una gran práctica para su aprendizaje. Por otra parte, si se potencia la educación musical, se favorece la adquisición de las funciones ejecutivas que son las que permiten a los estudiantes desarrollar procedimientos de aprendizaje, como por ejemplo la percepción, la atención, la memoria, entre algunas (Corral y Brull, 2006; Costa-Giomi, 2015; León, 2018; Nelson y DeBacker, 2008), asimismo normalmente se contribuye a mejorar los rendimientos académicos (Lacasta, 2017; Schellenberg et al., 2007).

Este trabajo se ha efectuado en un centro en el que se está aplicando la metodología didáctica de IM, se trata del Centro Integrado de Música 'Padre Antonio Soler' que comprende las etapas de Educación Primaria y Secundaria en el que los alumnos estudian de forma simultánea las enseñanzas musicales y las ordinarias. En este estudio se ha valorado la conexión que hay entre las IM y sus rendimientos académicos tanto en las asignaturas generales como en las musicales en estudiantes de Primero de Educación Secundaria Obligatoria. En estas Escuelas Integradas de Música se ha constatado que los alumnos que demuestran una buena práctica y habilidad en sus procesos de aprendizaje suelen tener rendimientos positivos debido a su disciplina para trabajar de forma coordinada en la enseñanza ordinaria y en la musical (Moreno et al., 2011; Reyes, 2011; Roden, 2014). 
Del mismo modo que los centros ordinarios, preferentes y de educación especial, etc., las Escuelas Integradas de Música constituyen otra clase de instituciones educativas, en las que los estudiantes cursan de modo concurrente la escolaridad del programa educativo ordinario y el musical. Esta clase de instituciones integradas nacieron con la Ley Orgánica de Ordenación General del Sistema Educativo (LOGSE, 1990), pero su puesta en funcionamiento fue compleja a nivel educativo en las etapas de Educación Secundaria Obligatoria y Bachillerato porque era complicado combinar las dos enseñanzas a escala organizativa y curricular (Ibeas, 2016; Lemes, 2000).

Esta investigación se ha realizado en un grupo de estudiantes de Primero de ESO debido a que era un buen curso porque están estudiando para acceder a las enseñanzas profesionales musicales, y para ello deben hacer un examen para promocionar al siguiente grado musical. Estos alumnos eran buenos aspirantes para analizar su consecución de capacidades y habilidades que les ayudaban a lograr los objetivos académicos establecidos, entre otros, y por su adiestramiento musical alcanzan un buen nivel de autonomía y de gestión para su trabajo escolar. Asimismo, debido a la exigencia que poseen estos alumnos al tener que tocar diariamente el instrumento elegido, adquieren una mayor capacidad para realizar sus tareas lo que mejora su motivación para compaginar las dos enseñanzas y, adicionalmente, su ejercitación rutinaria en la modalidad musical potencia en ellos su compromiso con su estudio personal (Hansen et al., 2013; Matthews, 2011; Portowitz et al., 2009).

Para la recogida de datos se han realizado exámenes psicométricos relacionados con el grado de inteligencia de los estudiantes, que ha sido medida de forma general y por factores, con su nivel de funcionamiento en IM y con sus competencias para la música. La finalidad de este estudio es analizar el nivel de adquisición de determinadas capacidades por parte de estos alumnos por estar en un enfoque educativo distinto muy rígido, por tener que trabajar bastante y por al estudiar simultáneamente ambas modalidades educativas, la general (Lengua, Inglés, Educación Física, Matemáticas, Ciencias Sociales y Ciencias Naturales) y la musical (lenguaje musical, orquesta, coro, banda, etc.). Al fin y al cabo, estos alumnos tienen que lograr los objetivos académicos de las dos enseñanzas para lograr pasar de curso y de grado musical, ya que solamente pueden suspender dos asignaturas en el modelo ordinario y una en el musical como máximo.

El nexo entre las facultades intelectuales y la competencia musical es complicado de calcular ya que participan otros elementos como la motivación y la gestión del tiempo a invertir para el ejercicio musical, además de la tenacidad y los resultados logrados a través de ella. La formación musical favorece la creatividad, aumenta la autoestima, fortalece la capacidad interpersonal y, por otro lado, facilita el aprendizaje en el área de Lengua, de Matemáticas y en el campo de la inteligencia visoespacial (Costa-Giomi, 2015). 
Muñoz (2013) puso de manifiesto que el entrenamiento musical tiene un impacto directo sobre el desarrollo de la inteligencia emocional, sobre la capacidad de gestión de las emociones y, en general, sobre la conducta. Asimismo, descubrió que el ejercicio musical a través de un instrumento facilita la mejora de las competencias intrapersonales y favorece el rendimiento escolar en otras materias. Por otra parte, Lázaro (2015) encontró que los alumnos que tienen mayor pericia musical suelen alcanzar mejores resultados en las capacidades cognitivas, en cambio, los estudiantes que demostraron no tener habilidad musical no obtuvieron buenas puntuaciones en inteligencia.

El objetivo de esta investigación es comprobar, en la muestra elegida (grupo de alumnos de $1^{\circ}$ de la ESO), la relación entre:

- El grado de inteligencia general, factorial y el nivel de I.M.

- Las ocho inteligencias múltiples.

- Los rendimientos académicos en las asignaturas ordinarias y en las de la modalidad musical.

- El nivel de hábito de estudio y los resultados académicos en las asignaturas ordinarias y en las de la modalidad musical.

- Elección de instrumento musical por cada estudiante y los rendimientos en las asignaturas ordinarias y en las de modalidad musical.

\section{MÉTODO}

\section{Participantes}

La muestra elegida está formada por veintiocho estudiantes de $1^{\circ}$ de la ESO de un Centro Integrado de Música, que están cursando de modo paralelo la escolaridad ordinaria y la modalidad musical. A pesar de parecer un grupo pequeño para la realización del estudio, sin embargo, se trata de una muestra incidental que tiene un fuerte peso científico, ya que la proporción de estudiantes de ESO que se forman en Escuelas Integradas de Música es muy reducida, porque en la comunidad madrileña solamente hay 2 Centros de esta clase, que son el Padre Antonio Soler, sito en San Lorenzo del Escorial y el Federico Moreno Torroba, sito en Madrid capital, lo que representa un valor inferior al $1 \%$ de la totalidad. Los datos extraídos han sido muy útiles para estudiar la conexión entre las variables del estudio, por un lado, entre la inteligencia general, la factorial y las IM y, por otro lado, entre los resultados académicos en las asignaturas musicales y en las generales de ESO. 


\section{Instrumentos}

En este estudio se han tenido en cuenta las siguientes variables que han intervenido en la estimación:

La Inteligencia general y la factorial que está formada por la inteligencia verbal y la no verbal que se han medido como variables cuantitativas discretas.

I.M de los alumnos de una Escuela Integrada de Música que se han medido como variables cuantitativas discretas y se han evaluado con el respectivo cuestionario.

Resultados académicos en las asignaturas ordinarias (Matemáticas, Lengua, Inglés, Ciencias Naturales, Ciencias Sociales) y en las musicales (Lenguaje Musical, Coro, Instrumento, Banda, etc.) que se han medido como variables cuantitativas continuas.

Para medir el grado de inteligencia se ha utilizado el test de Inteligencia general y factorial de Yuste (1991), que está compuesto por 70 ítems y valora inteligencia general, razonamiento lógico, contenidos verbales, numéricos y espaciales.

Para realizar la evaluación sobre el modo de organizar el estudio y el trabajo escolar se ha utilizado el Cuestionario de técnicas y hábitos de estudio (IHE) de Pozar (1994), que está formado por 90 ítems, que miden los hábitos de estudio y de trabajo escolar, a través de 4 escalas claves que proporcionan datos sobre los condicionantes del ambiente para estudiar, de la organización del estudio, del uso de los medios materiales, de la adquisición de contenidos y consta de un parámetro que mide el grado de sinceridad.

Finalmente, para obtener datos sobre nivel de adquisición de las IM de los estudiantes se les aplicó el Cuestionario de IMCUIM (Aliaga et al., 2012) que está formado por 90 preguntas que valoran las 8 IE: la Inteligencia musical; la inteligencia linguiística; la Inteligencia lógico-matemática; la Inteligencia visoespacial; la Inteligencia kinestésico-corporal; la Inteligencia naturalista y la Inteligencia emocional (compuesta por la interpersonal y la intrapersonal).

Para termina la investigación, se recopilaron y estudiaron los resultados académicos, tanto de las asignaturas ordinarias (Matemáticas, Lengua, Inglés, Ciencias Naturales, Ciencias Sociales) como de las musicales (Instrumento, Lenguaje Musical, Banda y Coro), en las dos evaluaciones centrales del curso, de los estudiantes.

\section{Procedimiento}

Este trabajo se ha realizado en diversas sesiones en las que se aplicaron pruebas a los estudiantes para recoger los datos acerca de las variables de estudio que permitieron llevar a cabo un análisis descriptivo y correlacional entre ellas. Para hacerlo, se solicitó permiso a la Jefa de Estudios y al Departamento de Orientación, una vez aceptada el trabajo por ambos, se pidió por escrito una autorización a los padres y madres de los estudiantes para poder suministrar las distintas pruebas para la recogida de 
datos y los cuestionarios para evaluar sus competencias, habilidades y actitudes. Una vez que los padres respondieron a la petición se comenzó el trabajo de investigación con el alumnado. Los primeros cuestionarios que se aplicaron fueron el de IM y el de inteligencia general y factorial y, seguidamente, se aplicó el cuestionario de hábitos de estudio. Finalmente, se solicitaron al tutor del grupo las calificaciones de los resultados académicos de dos de las tres evaluaciones. Se llevaron a cabo las pruebas estadísticas y se hizo un análisis de los descriptivos de frecuencias y las correlaciones entre las variables y a partir de los resultados se extrajeron las correspondientes conclusiones.

\section{RESULTADOS}

\section{Búsqueda bibliográfica}

En este apartado, se realiza la interpretación de los resultados extraídos, cuyos valores más representativos están expuestos en las tablas 1, 2, 3, 4 y 5 siguientes.

Se ha constatado que, en general, los rendimientos académicos de los estudiantes en las asignaturas han sido óptimos en ambas modalidades, la general y la musical y se ha comprobado que los alumnos de la muestra tienen destrezas destacadas en algunas de las IM y que desarrollan unos hábitos de estudio específicos que les permite compaginar ambas modalidades educativas.

Entre los resultados hay que destacar los siguientes:

En la tabla 1 se puede observar que existe una relación significativa de la inteligencia general con la no verbal (0.911) y con la verbal (0.842); y el índice de correlación entre la no verbal y la verbal que es de 0.556 .

Tabla 1. Correlaciones entre inteligencia general, verbal y no verbal

\begin{tabular}{|c|c|c|c|c|}
\hline & & $\begin{array}{c}\text { Inteligencia } \\
\text { general }\end{array}$ & $\begin{array}{c}\text { Inteligencia } \\
\text { no verbal }\end{array}$ & $\begin{array}{c}\text { Inteligencia } \\
\text { verbal }\end{array}$ \\
\hline \multirow{3}{*}{$\begin{array}{l}\text { Inteligencia } \\
\text { general }\end{array}$} & Pearson Correlation & 1 & $.911^{* *}$ & $.842^{* *}$ \\
\hline & Sig. (2-tailed) & & .000 & .000 \\
\hline & $\mathrm{N}$ & 27 & 27 & 27 \\
\hline \multirow{3}{*}{$\begin{array}{l}\text { Inteligencia } \\
\text { no verbal }\end{array}$} & Pearson Correlation & $.911^{* *}$ & 1 & $.556^{* *}$ \\
\hline & Sig. (2-tailed) & .000 & & .003 \\
\hline & $\mathrm{N}$ & 27 & 27 & 27 \\
\hline \multirow{3}{*}{$\begin{array}{l}\text { Inteligencia } \\
\text { verbal }\end{array}$} & Pearson Correlation & $.842^{* *}$ & $.556^{* *}$ & 1 \\
\hline & Sig. (2-tailed) & .000 & .003 & \\
\hline & $\mathrm{N}$ & 27 & 27 & 27 \\
\hline
\end{tabular}

En cuanto a la relación entre las IM y la inteligencia general, como puede percibirse en la tabla 2 , es significativa con la inteligencia lógico-matemática (0.531) y con la interpersonal (0.395). Asimismo, hay relación entre la inteligencia lógicomatemática y la inteligencia verbal (0.644). 
Tabla 2. Correlaciones entre inteligencia general e IE

\begin{tabular}{lcccccccc}
\hline & $\begin{array}{c}\text { Intelig. } \\
\text { general }\end{array}$ & $\begin{array}{c}\text { Inteligencia } \\
\text { verbal- } \\
\text { lingüística }\end{array}$ & $\begin{array}{c}\text { Inteligencia } \\
\text { lógico- } \\
\text { matemática }\end{array}$ & $\begin{array}{c}\text { Inteligencia } \\
\text { espacial }\end{array}$ & $\begin{array}{c}\text { Inteligencia } \\
\text { física- } \\
\text { cinestésica }\end{array}$ & $\begin{array}{c}\text { Inteligencia } \\
\text { músical }\end{array}$ & $\begin{array}{c}\text { Inteligencia } \\
\text { intrapersonal }\end{array}$ & $\begin{array}{c}\text { Inteligencia } \\
\text { interpersonal }\end{array}$ \\
\hline $\begin{array}{l}\text { Inteligencia } \\
\text { general }\end{array}$ & 1 & -.029 & $.531^{* *}$ & .100 & -.261 & -.366 & -.118 & $-.395^{*}$ \\
\cline { 2 - 9 } & & .887 & .004 & .618 & .189 & .060 & .556 & .042 \\
\hline
\end{tabular}

En la tabla 3, se puede percibir que el nivel de interrelación entre las IM es bastante considerable, cuyos resultados son los siguientes:

El grado de correlación de la inteligencia lingüística con la intrapersonal es de 0.408 y con la viso-espacial es de 0.385 . El grado de correlación de la inteligencia lógico-matemática con la interpersonal es de 0.477 y con la viso-espacial es de 0.502 . El grado de correlación entre la inteligencia quinestésica con la interpersonal es de 0.389 y con la musical es de 0.410 . El grado de correlación entre la inteligencia interpersonal y la intrapersonal es de 0.495 .

Tabla 3. Correlaciones entre inteligencias múltiples

\begin{tabular}{lccccccc}
\hline & $\begin{array}{c}\text { Inteligencia } \\
\text { verbal- } \\
\text { lingǘstica }\end{array}$ & $\begin{array}{c}\text { Inteligencia } \\
\text { lógico- } \\
\text { matemática }\end{array}$ & $\begin{array}{c}\text { Inteligencia } \\
\text { espacial }\end{array}$ & $\begin{array}{c}\text { Inteligencia } \\
\text { física- } \\
\text { cinestésica }\end{array}$ & $\begin{array}{c}\text { Inteligencia } \\
\text { musical }\end{array}$ & $\begin{array}{c}\text { Inteligencia } \\
\text { intrapersonal }\end{array}$ & $\begin{array}{c}\text { Inteligencia } \\
\text { interpersonal }\end{array}$ \\
\hline $\begin{array}{l}\text { Inteligencia } \\
\text { verbal-lingǘstica }\end{array}$ & 1 & .250 & $.385^{*}$ & .243 & .312 & $.408^{*}$ & .163 \\
\hline $\begin{array}{l}\text { Inteligencia } \\
\text { lógico- } \\
\text { matemática }\end{array}$ & .250 & 1 & $.502^{* *}$ & .072 & .045 & -.219 & $-477^{*}$ \\
\hline $\begin{array}{l}\text { Inteligencia } \\
\text { espacial }\end{array}$ & $.385^{*}$ & $.502^{* *}$ & 1 & .116 & .026 & .035 & -.236 \\
\hline $\begin{array}{l}\text { Inteligencia } \\
\text { física-cinestésica }\end{array}$ & .243 & .072 & .116 & 1 & $.410^{*}$ & .055 & $.389^{*}$ \\
\hline $\begin{array}{l}\text { Inteligencia } \\
\text { músical }\end{array}$ & .312 & .045 & .026 & $.410^{*}$ & 1 & -.029 & .288 \\
\hline $\begin{array}{l}\text { Inteligencia } \\
\text { intrapersonal }\end{array}$ & $.408^{*}$ & -.219 & .035 & .055 & -.029 & 1 & $.495^{* *}$ \\
\hline $\begin{array}{l}\text { Inteligencia } \\
\text { interpersonal }\end{array}$ & .163 & $-.477^{*}$ & -.236 & $.389^{*}$ & .288 & $.495^{* *}$ & 1 \\
\hline
\end{tabular}

En el análisis de los valores medios de los rendimientos académicos de los estudiantes se ha verificado que su media ha sido más baja en las asignaturas ordinarias, cuyo valor ha sido de 6.3, que, en las asignaturas musicales, cuyo valor ha sido de 7.5.

Por otra parte, el índice de correlación entre las calificaciones de las asignaturas ordinarias y las musicales ha sido de 0.694 , por consiguiente, en el presente trabajo los estudiantes que obtienen buenas notas en las materias musicales suelen adquirirlas también en las generales y a la inversa.

Los valores del índice de correlación entre las calificaciones de los estudiantes y sus hábitos de trabajo son los siguientes (véase la Tabla 4):

En las asignaturas de carácter general se relacionan de manera relevante con los medios de asimilación (0.678) y con los materiales para estudiar (0.428). 
En las asignaturas de música tienen un grado de correlación mayor con la capacidad de asimilación (0.521) y con los materiales utilizados para el estudio (0.398). Los estudios incluidos nos proporcionan un total de 13.589 pacientes con hemofilia A severa de los cuales 6.181 se encuentran bajo tratamiento profiláctico específicamente definido.

Tabla 4. Relación entre hábitos de estudio y rendimientos académicos

\begin{tabular}{lccccccc}
\hline & $\begin{array}{c}\text { Hábito } \\
\text { estudio- } \\
\text { ambiente }\end{array}$ & $\begin{array}{c}\text { Hábito } \\
\text { estudio- } \\
\text { planificación }\end{array}$ & $\begin{array}{c}\text { Hábito } \\
\text { estudio- } \\
\text { materiales }\end{array}$ & $\begin{array}{c}\text { Hábito } \\
\text { estudio- } \\
\text { asimilación }\end{array}$ & $\begin{array}{c}\text { Hábito } \\
\text { estudio- } \\
\text { sinceridad }\end{array}$ & $\begin{array}{c}\text { Media } \\
\text { notas } \\
\text { asignaturas } \\
\text { musicales }\end{array}$ & $\begin{array}{c}\text { Media } \\
\text { notas } \\
\text { asignaturas } \\
\text { generales }\end{array}$ \\
\hline $\begin{array}{l}\text { Hábito estudio- } \\
\text { ambiente }\end{array}$ & 1 & $.527^{* *}$ & $.654^{* *}$ & $.474^{*}$ & .219 & .095 & .219 \\
\hline $\begin{array}{l}\text { Hábito estudio- } \\
\text { planificación }\end{array}$ & $.527^{* *}$ & 1 & $.724^{* *}$ & $.691^{* *}$ & -.012 & .348 & $.471^{*}$ \\
\hline $\begin{array}{l}\text { Hábito estudio- } \\
\text { materiales }\end{array}$ & $.654^{* *}$ & $.724^{* *}$ & 1 & $.605^{* *}$ & .065 & $.398^{*}$ & $.428^{*}$ \\
\hline $\begin{array}{l}\text { Hábito estudio- } \\
\text { asimilación }\end{array}$ & $.474^{*}$ & $.691^{* *}$ & $.605^{* *}$ & 1 & -.234 & $.521^{* *}$ & $.678^{* *}$ \\
\hline $\begin{array}{l}\text { Hábito estudio- } \\
\text { sinceridad }\end{array}$ & .219 & -.012 & .065 & -.234 & 1 & -.076 & -.131 \\
\hline $\begin{array}{l}\text { Media notas } \\
\text { asignaturas } \\
\text { musicales }\end{array}$ & .095 & .348 & $.398^{*}$ & $.521^{* *}$ & -.076 & 1 & $.694^{* *}$ \\
\hline $\begin{array}{l}\text { Media notas } \\
\text { asignaturas } \\
\text { generales }\end{array}$ & .219 & $.471^{*}$ & $.428^{*}$ & $.678^{* *}$ & -.131 & $.694^{* *}$ & 1 \\
\hline
\end{tabular}

Por último, como se refleja en la tabla 5, en el estudio de las notas obtenidas por los alumnos en función de los instrumentos elegidos se percibe que los que obtienen mejores resultados en los rendimientos, tanto musicales como generales, son los que tocan instrumentos de aire, cuyas medias son 7.87 y de 6.96, respectivamente. Y los que logran peores resultados son los que tocan instrumentos de percusión, con medias de 6.8 y 5.4, respectivamente.

Tabla 5. Medias rendimientos musicales y generales y elección de instrumento musical

\begin{tabular}{|c|c|c|c|}
\hline $\begin{array}{l}\text { Instrumentos } \\
\text { musicales }\end{array}$ & & $\begin{array}{c}\text { Media notas } \\
\text { asignaturas musicales }\end{array}$ & $\begin{array}{c}\text { Media notas } \\
\text { asignaturas generales }\end{array}$ \\
\hline \multirow{3}{*}{ Cuerda } & Media & 7.440 & 5.720 \\
\hline & $\mathrm{N}$ & 10 & 10 \\
\hline & DT & 1.0265 & 1.6739 \\
\hline \multirow{3}{*}{ Aire } & Media & 7.877 & 6.969 \\
\hline & $\mathrm{N}$ & 13 & 13 \\
\hline & DT & .8526 & 1.2671 \\
\hline \multirow{3}{*}{ Percusión } & Media & 6.800 & 5.400 \\
\hline & $\mathrm{N}$ & 3 & 3 \\
\hline & DT & 1.0000 & .9165 \\
\hline \multirow{3}{*}{ Total } & Media & 7.543 & 6.300 \\
\hline & $\mathrm{N}$ & 28 & 28 \\
\hline & DT & .9527 & 1.5611 \\
\hline
\end{tabular}




\section{DISCUSIÓN Y CONCLUSIONES}

A continuación, se exponen las conclusiones que se han extraído en el presente trabajo tras el análisis y descripción del funcionamiento de las variables en la muestra. Por un lado, se percibe que los estudiantes cuentan con una destreza musical óptima, y además demuestran tener una buena capacidad intrapersonal e interpersonal, porque sus interacciones con los demás son positivas, apropiadas y demuestran interés por sus compañeros, lo que facilita que exista un clima agradable en la clase que contribuye al desarrollo adecuado del proceso de enseñanza-aprendizaje (en adelante ea) tanto en el área musical como en la ordinaria. En concreto, a la hora de preparar conciertos tienen que ensayar de manera grupal y se apoyan y se ayudan entre ellos; inclusive en las audiciones, pese a que son actuaciones en solitario, colaboran unos con otros observándose una gran empatía entre sí. Asimismo, sobresalen en la habilidad intrapersonal, ya que demuestran una gran capacidad para conocer y entender sus propios sentimientos y emociones.

En las inteligencias que obtienen puntuaciones más bajas es en la verbal y en la espacial; y las calificaciones intermedias se dan en la lógico-matemática y la cinestésica. Sin embargo, Andreu y Godall (2012) comprobaron que los alumnos que estudian música tenían mayor destreza en el ámbito matemático y en el socio-natural y coinciden con nuestro trabajo en que destacan en las habilidades interpersonales.

El nivel de inteligencia general en estos alumnos varía bastante, algunos tienen un grado muy alto, pero en general están por encima de la media, pero sin excesos. Por lo tanto, no es un requisito indispensable para estudiar las dos enseñanzas el poseer superdotación, sino que es suficiente contar con un nivel medio alto (BarrientosFernández et al., 2019). En el estudio de Lacasta (2017) se llegó a las mismas conclusiones y es que comprobaron que los alumnos con un buen nivel musical tenían un buen rendimiento intelectual en las enseñanzas generales.

Se ha observado que muchos estudiantes tienen buenas calificaciones en las asignaturas musicales ya que están muy estimulados para estudiarlas y emplean mucho tiempo en tocar el instrumento que han elegido y disfrutan con ello. Sin embargo, algunos de ellos obtienen peores resultados en las materias ordinarias y, por tanto, es importante que sus tutores estén pendientes de sus rendimientos teniendo en cuenta los criterios para pasar de curso y de grado en las enseñanzas musicales. Costa-Giomi (2015) comprobó que el entrenamiento y adiestramiento en la disciplina musical aumenta la motivación para trabajar en otras áreas académicas escolares, fortalece la capacidad para relacionarse con los demás y refuerza la habilidad creativa. Koelsch (2012) detectó que los profesionales de la música al tocar un instrumento o generar sonidos musicales suscitan sentimientos y emociones. 
Por otra parte, Corral y Brull (2006) demuestran que la ejercitación en la música potencia el grado de autoconocimiento y autoestima, además refuerza la capacidad perceptiva y atencional, asimismo, comprobaron que sus estudiantes mejoran sus resultados académicos y adquieren un comportamiento más benévolo con ellos mismos y con los demás y adoptan una actitud de colaboración con sus compañeros. Nelson y DeBacker (2008) verificaron que hay una fuerte relación entre la lectura y la música, porque las dos precisan de una fuerte motivación personal, de sosiego emocional y de serenidad espiritual.

Asimismo, se ha constatado que los estudiantes que cursan las dos enseñanzas de forma simultánea (la musical y la ordinaria) han adquirido una buena capacidad para programar sus métodos de trabajo y para establecerse unos hábitos de estudio apropiados que les ayuden a simultanear sus tareas escolares con el ejercicio musical diario. Hansen et al. (2013) sostienen que el trabajo musical contribuye a mejorar la voluntad de los estudiantes para realizar su estudio y sus actividades. Portowitz et al. (2009) descubrieron que, en una muestra de estudiantes, con y sin formación musical, existían discrepancias entre las estrategias que utilizaban para realizar su proceso de adquisición de conocimientos y su capacidad intelectual. De igual manera, Matthews (2011) destaca que el entrenamiento musical ayuda a fortalecer otras competencias como la capacidad para planificarse, la constancia en el trabajo, la adquisición de estrategias de concentración, la motivación personal, la autorregulación y la autodisciplina.

Se ha corroborado que estos alumnos consiguen rendimientos académicos positivos en las modalidades tanto musical como ordinaria, de igual forma, Schellenberg et al. (2007) comprobaron que el ejercicio musical potencia el proceso de e-a y, en consecuencia, mejora los resultados académicos de otras materias. Moreno et al. (2011), en concreto, vieron que el ejercicio de un sistema musical de formación aumenta el rendimiento en la asignatura de Lengua. Análogamente, Roden (2014) encontró en una muestra que los alumnos que estudiaron en un programa de formación musical sacaron mejores notas que los que no lo habían hecho. De igual manera, Reyes (2011) confirmó que los Centros de Música dan la oportunidad a los estudiantes para adquirir habilidades para aprender $\mathrm{y}$, en consecuencia, potencian su capacidad intelectual en otras asignaturas, como por ejemplo en Matemáticas, Lengua; Ciencias Sociales, etc. Por otra parte, debido a la gran exigencia que tienen los alumnos en el área musical se sienten obligados a organizarse mejor y a planificar su tiempo de estudio en las materias ordinarias y, de ese modo, lograr un mejor rendimiento. No obstante, en ocasiones, es complicado medir el grado de correlación entre la habilidad cognitiva y la musical ya que intervienen otros elementos como la motivación para planificarse el tiempo de dedicación a la actividad musical, la constancia en el trabajo y los resultados logrados con esta actividad. 
Por otra parte, hemos comprobado que estos alumnos requieren hacer un elevado esfuerzo, desarrollar una gran autodisciplina y planificar su trabajo de forma adecuada porque necesitan contar con períodos de tiempo concretos para las dos formaciones. Asimismo, estos estudiantes tienen que ser conscientes de que los objetivos curriculares generales son los mismos que los de los alumnos que estudian solo en la modalidad ordinaria, pero añadiendo los objetivos de la enseñanza musical. Por ello, es clave que posean una buena capacitación en inteligencia musical (tales como tener ritmo, buen oído y percepción auditiva), una adecuada memoria, capacidad de trabajo, habilidades intrapersonales e interpersonales, etc. En consecuencia, para ser capaces de estudiar en una Escuela Integrada de Música con éxito, los estudiantes deben tener algunos rasgos y actitudes como un nivel intelectual apropiado, buenas condiciones físicas, perseverancia y compromiso, voluntad, conocimientos y procedimientos musicales, etc. En conclusión, estos alumnos no son muy distintos a otros, lo que les diferencia es su dedicación a la música, desde tercero de Primaria (8 años) a segundo de Bachillerato, su capacidad de autodisciplina y de autonomía y su especial sensibilidad por el arte.

Como síntesis de esta investigación, se ha verificado que el entrenamiento musical favorece en los alumnos la consecución de otras destrezas como la inteligencia emocional (interpersonal e intrapersonal), asimismo mejora sus calificaciones académicas de forma global. Para corroborar los resultados obtenidos, se propone realizar un estudio de mayor alcance en el que se amplíe la muestra de estudio, incluyendo en ella sujetos de otras Escuelas Integradas de Música españolas, para obtener datos más representativos y extrapolables a la población.

Por último, se plantea realizar un estudio para comparar los datos acerca de capacidad de los estudiantes para organizar su trabajo y sus IM. Para ello, se seleccionará una muestra con alumnos de primero de Educación Secundaria Obligatoria, tanto de Centros Ordinarios como de Escuelas Integradas de Música, con el fin de evaluar y contrastar sus resultados y poder confirmar si es ventajosa o no la enseñanza en dichas Escuelas Integradas, es decir, si se favorece la dedicación, el esfuerzo, la autonomía y la motivación de sus alumnos al cursar de forma concurrente ambas enseñanzas, la musical y la ordinaria.

\section{REFERENCIAS}

Aliaga, J., Ponce, C., Bulnes, M., Elizalde, R., Montgomery, W., Gutiérrez, V., y Torchiani, R. (2012). Las IE: evaluación y relación con el rendimiento en matemática en estudiantes del quinto año de secundaria de Lima Metropolitana. Revista de Investigación en Psicología, 15(2), 163-202. doi:10.15381/rinvp.v15i2.3699 
Andreu, M., y Godall-Castell, P. (2012). La importancia de la educación artística en la enseñanza obligatoria: la adquisición de las competencias básicas de Primaria en un centro integrado de música. Madrid: Ministerio de Educación. doi:10.12795/ie.2018.i89.05

Barrientos-Fernández, A., Sánchez-Cabrero, R., Arigita-García, A., Mañoso-Pacheco, L., Pericacho-Gómez, F.J., y Novillo-López, M. Á. (2019). Measurement of different types of intelligence (general, verbal vs. non-verbal, multiple), academic performance and study habits of secondary students at a Music Integrated Centre. Data in Brief, 10(25), 104-124. doi:10.1016/j.dib.2019.104124

Corral, M.A.B., y Brull, V.A. (2006). Las agrupaciones musicales como reforzadores del rendimiento escolar. Música y educación: Revista trimestral de Pedagogía Musical, 19(66), 33-50.

Costa-Giomi, E. (2015). The Long-Term Effects of Childhood Music Instruction on Intelligence and General Cognitive Abilities. Update: Applications of Research in Music Education, 33(2), 20-26. doi: 10.1177/8755123314540661

Ferrándiz, C., Prieto, M.D., Bermejo, M.R., y Ferrando, M. (2006). Fundamentos psicopedagógicos de las IE. Revista Española de Pedagogía, 233, 5-19.

Garagorri, X. (2007). Currículo basado en competencias: aproximación en estado de la cuestión. Aula de Innovación Educativa, 16(161), 47-55.

Gardner, H. (1983). Multiple Intelligences. Barcelona: Paidós.

Gardner, H. (2008). Las cinco mentes del futuro. Barcelona: Paidós.

Gardner, H. (2016). Estructuras de la mente: la teoría de las IE. Madrid: Fondo de cultura económica.

Guzmán, B., y Castro, S. (2017). Las IE en el aula de clases. Revistas de Investigación, 29(58), 5361.

Hansen, M., Wallentin, M., y Vuust, P. (2013). Working memory and musical competence of musicians and non-musicians. Psychology of Music, 41(6), 779-793. doi: $10.1177 / 0305735612452186$

Ibeas-López, M. (2016). Enseñanza-aprendizaje musical: una visión de los docentes de instrumento en los conservatorios profesionales (Tesis Doctoral) País Vasco: Universidad del País Vasco. doi: 10.22550/rep75-2-2017-03

Koelsch, S. (2012). Brain and Music. UK: Wiley and Blackwell.

Lacasta, R.K.A. (2017). Memoria de trabajo: relación con el entrenamiento musical y el rendimiento académico (Tesis Doctoral). Salamanca: Universidad Pontificia de Salamanca.

Lázaro, F.R. (2015). Relación de aptitudes musicales, intelectuales y rasgos de personalidad e identificación del talento musical en escolares de diez a doce años (Tesis Doctoral) Murcia: Universidad de Murcia.

Lemes, F.L. (2000). Los centros integrados de música: aportaciones para la construcción de una alternativa. Eufonía: Didáctica de la Música, 1(19), 8-20.

León, M. (2018). Neurodidáctica Musical y Procesos de Aprendizaje. Un enfoque para la Formación del Educador Musical. Revista Educación Superior y Sociedad (ESS), 19(19), $1-40$.

Ley Orgánica 1/1990, de 3 de octubre, de Ordenación General del Sistema Educativo. Boletín Oficial del Estado, España, 4 de octubre de 1990.

Ley Orgánica 2/2006, de 3 de mayo, de Educación. Boletín Oficial del Estado, España, 4 de mayo de 2006.

Ley Orgánica, 8/2013, de 9 de diciembre, para la Mejora de la Calidad Educativa. Boletín Oficial del Estado, España, 10 de diciembre de 2013. 
López, X.C.M. (2017). La práctica musical como estrategia metodológica para el desarrollo del pensamiento formal en ambientes escolares. Revista Publicando, 4(11), 764-781.

Matthews, M. (2011). 18 Benefits of Playing a Musical Instrument. Recuperado de: http://www.effectivemusicteaching.com/articles/directors/18-benefits-ofplaying-amusical-instrument/2011/

Moreno, S., Bialystok, E., Barac, R., Schellenberg, E.G., Cepeda, N.J., y Chau, T. (2011). Shortterm music trainig enhances verbal intelligence and executive function. Psychology of Music, 22(11), 1425-1433. doi: 10.1177/0956797611416999

Muñoz, E.A.C. (2013). El desarrollo de las competencias intrapersonales a través del aprendizaje de un instrumento musical. Fòrum de Recerca, 18, 423-440. doi:10.6035/forumrecerca.2013.28

Nelson, M., y De Backer, T. (2008). Achievement Motivation in Adolescents: The Role of PeerClimate and Best Friends. The Journal of Experimental Education, 76(2), 170-189. doi: 10.3200/jexe.76.2.170-190

Portowitz, A., Lichtenstein, O., Egorova, L., y Brand, E. (2009). Underlying mechanisms linking music education and cognitive modifiability. Research Studies in Music Education, 31(2), 107-128. doi: 10.1177/1321103x09344378

Pozar, F.F. (1994). IHE, Inventario de Hábitos de Estudio: manual. Madrid: TEA Ediciones.

Reyes, M.C. (2011). El rendimiento académico de los alumnos de primaria que cursan estudios artístico-musicales en la Comunidad Valenciana (Tesis Doctoral). Valencia: Universitat de València. doi: 10.23913/ride.v9i18.480

Roden, E. (2014). Does music training enhance working memory performance? Findings from a quasi-experimental longitudinal study. Phychology of Music, 42(2), 284-298. doi: $10.1177 / 0305735612471239$

Schellenberg, E.G., Nakata, T., Hunter, P.G., y Tamoto, S. (2007). Exposure to music and cognitive performance: tests of children and adults. Psychology of Music, 35(1), 5-19. doi: $10.1177 / 0305735607068885$

Vivas, B.N. (2017). Las IE como una estrategia didáctica para atender a la diversidad y aprovechar el potencial de todos los alumnos. Revista de Educación Inclusiva, 8(3), 121-136. doi:10.12795/ie.2017.i90.02

Yuste-Hernanz, C. (1991). Inteligencia general y factorial. Madrid: TEA Ediciones.

Recibido: 11 de enero de 2019 Recepción Modificaciones: 21 de enero de 2019 Aceptado: 4 de febrero de 2019 